\author{
Matei KUBINSCHI, PhD Student \\ E-mail: matei.kubinschi@bnro.ro \\ Dinu BARNEA, PhD Student \\ E-mail: dinu.barnea@bnro.ro \\ The Bucharest University of Economic Studies
}

\title{
GLOBAL VALUE CHAINS AND INDUSTRIAL PRODUCTION SPILLOVERS: WHERE DOES THE CEE REGION STAND?
}

\begin{abstract}
The present paper aims at developing a better understanding of how the industrial sectors in different countries influence each other and how developments on western markets impact the growth rates of emerging economies, with a focus on the CEE region. Applying a framework developed by Diebold and Yilmaz (2009), we assess bilateral spillovers between a large set of EU members' Industrial Production Indices and find that, after the downturn episode of 2009-2011, spillover effects have significantly grown in importance. Therefore, we estimate an Industrial Production Spillover Index and assess whether including the index in a BVAR forecasting framework can lead to an increase in forecast accuracy, for selected CEE states (the Czech Republic, Poland, Hungary and Romania). Using multiple calibration strategies to ensure robustness, we find an overall increase in forecasting accuracy for Hungary, followed by the Czech Republic and Romania, where the results are mostly positive in favor of including the index, but also depend on the choice of the calibration methodology. Conversely, the results for Poland show that the augmented model offers lower forecast accuracy, in all the considered cases. Our main policy recommendation is related to assessing whether information related to industrial sector spillovers is relevant in explaining real growth dynamics and potentially including this information in the overall framework for monitoring macroeconomic policy. The policy implications brought by spillover effects highlight certain fragilities of the CEE economies, which are especially vulnerable in case of a slowdown in industrial activity in the Western EU states.
\end{abstract}

Keywords: industrial production, spillovers, density forecasting, CEE, forecast evaluation.

\section{JEL Classification: E32, C11, C53}

\section{Introduction}

Global integration in international value-added chains has played a pivotal role in the development of the industrial sectors of CEE countries in the post-accession period. Coming into the century with a low level of competitiveness, based chiefly

DOI: $10.24818 / 18423264 / 53.1 .19 .06$ 


\section{Matei Kubinschi, Dinu Barnea}

on cheap labor force and other aspects of cost competitiveness, CEE economies have benefited considerably from the liberalization of cross-border trade and capital flows, which has made them more attractive for foreign investment and inclusion in global supply chains. Relocating the production of intermediate goods to developing countries enables transnational companies to enhance productivity and lower production costs by accessing the best available resources each country has to offer. Simultaneously, emerging economies benefit through the numerous externalities that these changes bring: the creation of jobs, economy-wide technology shocks or the adoption of business and managerial models by local companies.

Integration in the global chains has thus become the main pillar of the exportled growth experienced by emerging economies in the EU. The case of Romania is conclusive in this regard. In the 10 years since joining the EU in 2007, the economy grew by around $20 \%$, out of which the industrial sector has accounted for more than 23 percentage points and about half of the total exports in 2016. This was only possible against the backdrop of a constant shift from less complex, often labor-intensive sectors (textiles, food products and beverages, coke and refining) towards higher-tech and -value added operations in industries such as electrical machinery, motor vehicles and components or machinery and equipment.

Seeing as the great majority of these newly developed industrial sectors in the CEE economies are deeply involved in international global value added chains and depend decisively on the performance of other participants in the chain and the competitiveness of the final product as a whole, we attempt to develop a better understanding of how the industrial sectors in different countries influence each other and how developments on western markets impact the growth rates of emerging economies. Given the interconnectedness of these markets and assuming that spillovers are mostly directed from high income to emerging economies, such an indicator may offer an early warning system for looming crises threatening developing countries.

\section{Literature Review}

We draw from Yilmaz (2009) to create an index which measures the direction and intensity of spillovers between member states' industrial sectors. However, the study of spillovers dates back to the beginning of the 19990s. Engle et al. (1990) analyze instances of volatility clustering on the yen/dollar exchange rate and find them to be caused by volatility spillovers from the capital markets rather than by the arrival of new information.

King et al. (1994) examine the role of observable macroeconomic variables in explaining the covariances between markets. They conclude that only a small fraction of co-movements can be attributed to measurable variables, and they are mainly driven by unobservable variables or investor sentiment. They also find important differences between the pricing of risk on different capital markets, serving as proof for a significantly priced idiosyncratic risk and low integration of global stock markets. 
Global Value Chains and Industrial Production Spillovers: Where Does the CEE Region Stand?

Edwards and Susmel (2001) use data from a number of South American and Asian economies to analyze whether the increasing financial instability seen in these markets in the 90s share common patterns among countries. Their results show that high volatility episodes tend to be short-lived rather than manifesting a secular tendency and that the co-movements in volatility across countries are often statistically significant. They find strong regional correlations between the Latin American markets, which also tend to increase during high volatility periods due to international crises, but low spillovers from Asian towards American markets.

Diebold and Yilmaz (2012) employ a generalized vector autoregressive framework with order-invariant forecast variance decomposition to measure volatility spillovers between the U.S. stock, bond, foreign exchange and commodity markets. They too find that cross-market spillovers, although present, were rather limited before the global financial crisis, but intensified significantly after the collapse of Lehman Brothers in September 2008, most notably from the stock market towards the others. Diebold and Yilmaz (2009) measure linkages of asset returns and asset volatilities across 19 stock markets -7 developed and 12 emerging. They find that financial integration has led to a steadily increasing trend of return spillovers, and no trend but clear bursts (associated to crisis events) in volatility spillovers.

Based on the aforementioned work, Yilmaz (2009) develops a spillover index for business cycles across the G6 economies (excluding Canada from the G7 countries). His framework is based on decomposing the variance of VEC forecast errors in order to discern between internal shocks and spillover of shocks. The variables included in this system are the seasonally adjusted monthly industrial production indices for each of the 6 countries. He employs a rolling window approach to account for the time-variation of spillover effects. His results show that there are statistically significant business cycle spillovers across G6 countries, their intensity is indeed time-varying, and that the U.S. and Japan are the main sources of business cycle shocks towards the other countries.

We attempt to build a similar indicator for EU countries in order to identify the intensities and directions of pairwise spillover effects for industrial production indices. Under the assumption that the more developed countries in the region (higher up the value added chains) tend to be the source of spillovers towards the new member states, we employ the spillover index in the GDP forecast of some CEE economies. Thereby we test the predictive power of the spillover index by measuring whether the augmenting of a Bayesian VAR with the index is likely to enhance forecast performance.

\section{Methodology and dataset}

\subsection{Measuring Industrial Production Spillovers in the EU}

The aim of the present study is to assess industrial production spillovers between EU members, with a focus on the CEE region. Applying the DieboldYilmaz (2009) methodology, we estimate a multivariate VAR model containing 


\section{Matei Kubinschi, Dinu Barnea}

indices of industrial production for several EU countries and compute bilateral spillover indices as well as an aggregate Industrial Production Spillover Index, whose utility will be argued in the second part of the empirical analysis.

Starting from the general framework of a $p$-lag VAR model:

$$
\begin{gathered}
Y_{t}=\sum_{i=1}^{p} \Phi_{i} Y_{t-i}+\varepsilon_{t} \text {, with the } \mathrm{MA}(\infty) \text { representation } \\
Y_{t}=\sum_{i=0}^{\infty} A_{i} \varepsilon_{t-i}
\end{gathered}
$$

We compute variance decomposition measures in order to assess the importance of the $\mathrm{H}$-step-ahead forecast error variance of an industrial production index that is due to shocks in all the other indices. Following Yilmaz (2009), we overcome the disadvantages associated with the Cholesky decomposition ordering of the variables by using the GeneralisedForecast Error Variance Decomposition (GFEVD), defined by Koop, Pesaran and Potter (1996) and Pesaran and Shin (1998), which generates ordering invariant results. The authors define the generalized impulse response function and forecast error variance decompositions as follows:

$$
\gamma_{j}^{g}(h)=\frac{1}{\sqrt{\sigma_{j j}}} A_{h} \sum e_{j} \text { and } \theta_{i j}^{g}(H)=\frac{\sigma_{i i}^{-1} \sum_{h=1}^{H-1}\left(e_{i}^{\prime} A_{h} \sum e_{j}\right)^{2}}{\sum_{h=1}^{H-1}\left(e_{i}^{\prime} A_{h} \sum A_{h}^{\prime} e_{j}\right)^{2}}
$$

To compute the relative contributions to each country by itself and other countries, we construct a weighted average using the sum of all contributions:

$$
\tilde{\theta}_{i j}^{g}(H)=\frac{\theta_{i j}^{g}(H)}{\sum_{j=1}^{N} \theta_{i j}^{g}(H)}
$$

At this stage, it is straightforward to compute the spillover index using a similar intuition, but weighting all spillover contributions except each country's own to the total sum of relative contributions:

$$
S^{g}(H)=\frac{\sum_{\substack{i, j=1, i \neq j}}^{N} \tilde{\theta}_{i j}^{g}(H)}{\sum_{i, j=1}^{N} \tilde{\theta}_{i j}^{g}(H)} \cdot 100
$$

\subsection{Dataset and results of the spillover analysis}

We build the database used for estimating the Industrial Production Spillover Index using data from EU states from Western (Belgium, Denmark, Germany, Spain, France, Italy, the Netherlands, Portugal and the United Kingdom) and Central and Eastern (Bulgaria, the Czech Republic, Hungary, Austria, Poland, Romania, Slovenia and Slovakia) Europe. We use seasonally and calendar adjusted monthly data extracted from the Eurostat database, on a timeframe spanning from 2000 to the end of 2017. When analyzing the evolution in time, we can split the full sample into three separate episodes: the first one up until 2008 is characterized by a relatively wide interquartile range, potentially signaling the fact that industrial production indices were predominantly driven by individual heterogeneous factors rather than common factors and spillover effects. The second part is the crisis and 
Global Value Chains and Industrial Production Spillovers: Where Does the CEE Region Stand?

recovery episode, spanning from 2008 to 2011, where the global financial crisis led to significant decreases in production at a global level, leading to a synchronized downturn in most EU countries. The final part, starting from 2011 until the end of the sample, displays a much narrower interquartile range as compared to previous years. This in turn could highlight the fact that, as a result of a higher level of integration within global value chains, increases in industrial production, following increases in demand, in certain larger economies have led to expansions in other regions of the EU as well.

To confirm the aforementioned hypotheses, we estimate the VAR model containing all the Industrial Production Indices, following the methodology described in the previous section. We compute the GFEVD on a 10 step-ahead basis and compute the bilateral spillover measures, which we report in Table 1. When analyzing the results for the entire sample, we ascertain that roughly 31 to 58 percent of the overall forecast error variance is explained by each country's own dynamics, leaving the rest to be accounted for spillovers from other states. Turning to the bilateral spillovers, two main groups can be identified: the Western EU states and the CEE region. Nevertheless, CEE region dynamics are also influenced by the Western group: countries such as Romania, Poland, Hungary and the Czech Republic receive significant inward spillovers from Germany, France, Italy and Belgium (ranging from 1-2 to 8 percentage points). When aggregating, we find that in general the CEE countries receive fewer spillovers than their Western counterparts (49-60 pp. as compared to 42-69 pp., last column in Table 2).

Table 1. Bilateral spillovers between selected EU members, 2000-2017 (10 steps-ahead, 4 lags)

\begin{tabular}{|c|c|c|c|c|c|c|c|c|c|c|c|c|c|c|c|c|c|}
\hline & UK & GER & ESP & FRA & ITA & BEL & POR & NED & AUT & BUL & CZE & HUN & $\mathrm{POL}$ & ROM & SLO & SVK & $\begin{array}{l}\text { From } \\
\text { others }\end{array}$ \\
\hline UK & 49.3 & 4.1 & 2.1 & 5.9 & 5 & 4.9 & 5.7 & 2 & 1.3 & 0.9 & 4.5 & 3.6 & 3.4 & 1.4 & 4.4 & 1.3 & 51 \\
\hline GER & 2.6 & 33.7 & 1.1 & 8.9 & 7.9 & 7 & 2.2 & 1.2 & 3.2 & 3.9 & 7.7 & 5.1 & 5.6 & 1.9 & 5.3 & 2.8 & 66 \\
\hline ESP & 0.9 & 1.1 & 57.9 & 3.6 & 2.1 & 3.5 & 7.3 & 2.9 & 2.1 & 3.2 & 1.3 & 4.6 & 1.8 & 1 & 1.5 & 5.1 & 42 \\
\hline FRA & 3.1 & 6.8 & 1.9 & 34.7 & 7.3 & 9 & 4.6 & 2.6 & 3 & 3.6 & 5.6 & 4.1 & 8.1 & 1.4 & 2.1 & 2.2 & 65 \\
\hline ITA & 3.8 & 6.4 & 4.3 & 7.8 & 31.1 & 6.8 & 5.8 & 1.2 & 2.7 & 3.4 & 6.9 & 3.3 & 8.3 & 2 & 2.7 & 3.5 & 69 \\
\hline BEL & 4.4 & 5.8 & 2.5 & 9.4 & 8.2 & 41.2 & 1.6 & 0.4 & 4 & 4.9 & 4.3 & 5.2 & 4.3 & 0.8 & 1.8 & 1.1 & 59 \\
\hline POR & 3.8 & 2.8 & 3.2 & 3.4 & 4.3 & 5.7 & 43.1 & 6.1 & 2.1 & 4.3 & 4.9 & 1.7 & 2.7 & 5.2 & 2 & 4.6 & 57 \\
\hline NED & 1.2 & 1.1 & 3.2 & 4.7 & 2.9 & 2.3 & 11.5 & 55.5 & 1.7 & 0.6 & 3.2 & 1.6 & 2 & 4.7 & 1.6 & 2 & 44 \\
\hline AUT & 2 & 4.3 & 2.1 & 5.3 & 5.3 & 6.6 & 1.9 & 2.1 & 40.2 & 5.4 & 6.9 & 4.8 & 2.8 & 3.2 & 4.2 & 2.9 & 60 \\
\hline BUL & 3.6 & 4.2 & 3.1 & 5.8 & 4.3 & 7.3 & 1.8 & 1.1 & 4 & 40.3 & 6.4 & 2.2 & 7.6 & 3.8 & 1.5 & 3 & 60 \\
\hline CZE & 3.1 & 5.9 & 3.1 & 3.6 & 3.5 & 3.5 & 2.6 & 2.4 & 2.1 & 5 & 40 & 6.4 & 6.6 & 3.9 & 1.9 & 6.5 & 60 \\
\hline HUN & 3.5 & 4.4 & 4 & 5.5 & 3.9 & 5.5 & 2.3 & 2.6 & 5.3 & 1 & 5.1 & 43 & 4.3 & 3.1 & 4.2 & 2.4 & 57 \\
\hline POL & 2.4 & 3.4 & 1.1 & 6.6 & 8 & 5 & 2.3 & 1 & 2 & 5.8 & 8 & 4.5 & 43.3 & 3.7 & 2 & 0.8 & 57 \\
\hline ROM & 1.5 & 2.8 & 1.9 & 2.5 & 3.5 & 7.7 & 1.3 & 3.1 & 2.8 & 6 & 3.9 & 3.7 & 3.9 & 51.1 & 1.5 & 2.9 & 49 \\
\hline SLO & 2.3 & 6.2 & 2.4 & 2.8 & 4.9 & 2.8 & 2.7 & 1.4 & 3.5 & 1.4 & 5.7 & 6.7 & 2.5 & 1.5 & 48.5 & 4.7 & 51 \\
\hline SVK & 1.8 & 2.7 & 3.4 & 2.6 & 6.7 & 1.9 & 4.3 & 3.5 & 3.5 & 2 & 5.9 & 3.8 & 1.7 & 2.3 & 6 & 47.7 & 52 \\
\hline $\begin{array}{c}\text { Contrib. to } \\
\text { others }\end{array}$ & 40 & 62 & 39 & 78 & 78 & 80 & 58 & 34 & 43 & 51 & 80 & 61 & 66 & 40 & 43 & 46 & 899 \\
\hline $\begin{array}{c}\text { Contribution } \\
\text { incl. own }\end{array}$ & 89 & 96 & 97 & 113 & 109 & 121 & 101 & 89 & 84 & 92 & 120 & 104 & 109 & 91 & 91 & 93 & \\
\hline
\end{tabular}

Source: authors' estimation 
In order to obtain further insight on spillover dynamics with a focus on the third part of the sample, beginning with 2011, we re-estimate the model and restrict the timeframe to 2011-2017. The results of the subsample analysis are presented in Table 2.

Table 2. Bilateral spillovers between selected EU members, 2011-2017 (10 steps-ahead, 4 lags)

\begin{tabular}{|c|c|c|c|c|c|c|c|c|c|c|c|c|c|c|c|c|c|}
\hline & UK & GER & ESP & FRA & ITA & BEL & POR & NED & AUT & BUL & CZE & HUN & POL & ROM & SLO & SVK & $\begin{array}{c}\text { From } \\
\text { others }\end{array}$ \\
\hline UK & 13.6 & 12.1 & 2.5 & 8.4 & 5.6 & 1.9 & 6.2 & 2.9 & 2.7 & 6.3 & 13.2 & 7.3 & 2.2 & 3.2 & 2.7 & 9.2 & 86 \\
\hline GER & 8.2 & 13.1 & 4.1 & 8.6 & 4 & 3.2 & 4.4 & 5.7 & 4.2 & 7.3 & 10.4 & 7 & 1.9 & 3.1 & 8.4 & 6.3 & 87 \\
\hline ESP & 3.2 & 5.5 & 19.2 & 4 & 8.4 & 8.6 & 3.8 & 3.1 & 6.6 & 6.7 & 9.9 & 1.3 & 2.5 & 5.5 & 3.8 & 7.8 & 81 \\
\hline FRA & 9.5 & 7.3 & 5.4 & 10.3 & 5.8 & 3.8 & 1.9 & 3.2 & 7.8 & 4.5 & 9.6 & 8.7 & 1.8 & 5.5 & 3.6 & 11.2 & 90 \\
\hline ITA & 2.9 & 6.4 & 7.5 & 3.4 & 9.5 & 3 & 6.7 & 2.9 & 9.1 & 9.6 & 9.6 & 3.2 & 3.2 & 5.5 & 0.8 & 16.7 & 91 \\
\hline BEL & 7.9 & 8.9 & 3.3 & 4.8 & 7.1 & 7.8 & 5.3 & 1.2 & 4.6 & 4.3 & 14.9 & 5.2 & 1.8 & 6.4 & 1.9 & 14.5 & 92 \\
\hline POR & 3.5 & 6.8 & 5.2 & 5.5 & 3.5 & 4.6 & 13.3 & 3.2 & 3 & 11.1 & 11.4 & 4.5 & 2.2 & 4.3 & 4 & 13.8 & 87 \\
\hline NED & 3.2 & 6.4 & 4.1 & 11.3 & 3.5 & 2.8 & 8.2 & 10.6 & 1.5 & 7.8 & 10 & 9.9 & 3.1 & 4.7 & 4.6 & 8.1 & 89 \\
\hline AUT & 4.1 & 6.3 & 3.2 & 5.2 & 6.8 & 2.9 & 6 & 4.1 & 9.9 & 9.7 & 8.2 & 7 & 3.8 & 9.2 & 1 & 12.6 & 90 \\
\hline BUL & 3.7 & 7.2 & 3.3 & 6.9 & 3.2 & 6.6 & 3.8 & 10.4 & 3.1 & 22.8 & 9.9 & 5.7 & 2.6 & 3.2 & 3 & 4.6 & 77 \\
\hline CZE & 6.4 & 9.7 & 3.1 & 11.5 & 5.3 & 1.9 & 6.6 & 5 & 1.7 & 5.1 & 19.2 & 7.5 & 1 & 1 & 7.8 & 7.2 & 81 \\
\hline HUN & 4.5 & 8.3 & 4.7 & 8.8 & 3.7 & 2 & 8.1 & 7.5 & 3.5 & 9.1 & 5.8 & 12.2 & 2.5 & 7.3 & 3.4 & 8.5 & 88 \\
\hline $\mathrm{POL}$ & 4.6 & 9 & 3.7 & 5.2 & 3.4 & 2.6 & 6.4 & 2.8 & 5.8 & 10.8 & 9.7 & 5 & 8.6 & 7 & 3.4 & 12.1 & 91 \\
\hline ROM & 4.9 & 10.2 & 3 & 6.6 & 2.5 & 3.8 & 5.3 & 7.7 & 3.4 & 6.3 & 10.1 & 5.4 & 4.4 & 8.4 & 10.5 & 7.4 & 92 \\
\hline SLO & 1.7 & 6 & 7.5 & 8.7 & 11.9 & 1.8 & 5.7 & 4.5 & 5.1 & 3.9 & 7.7 & 8.4 & 2.3 & 1.6 & 17.5 & 5.8 & 82 \\
\hline SVK & 3.4 & 6.5 & 6.1 & 4.9 & 4.1 & 1.4 & 8.7 & 2.1 & 2.1 & 11.2 & 12.2 & 3.3 & 2.1 & 5.4 & 4.6 & 21.9 & 78 \\
\hline $\begin{array}{c}\text { Contrib. to } \\
\text { others }\end{array}$ & 72 & 117 & 67 & 104 & 79 & 51 & 87 & 67 & 64 & 114 & 153 & 90 & 37 & 73 & 63 & 146 & 1382 \\
\hline $\begin{array}{l}\text { Contribution } \\
\text { incl. own }\end{array}$ & 85 & 130 & 86 & 114 & 88 & 59 & 101 & 77 & 74 & 136 & 172 & 102 & 46 & 81 & 81 & 168 & \\
\hline
\end{tabular}

Source: authors' estimation

In this case, the results paint a completely different picture: individual contributions (diagonal elements) now play a much smaller part in explaining overall dynamics, partially confirming the fact that spillovers from other countries have become increasingly important in recent years. This effect is especially strong in the CEE region, with own contribution ranging from about 8 percentage points in Romania and Poland to around 20 percentage points in the Czech Republic. Moreover, we observe a significant increase in spillovers from Germany, France, Portugal and the Netherlands which now account, in some cases, for almost double the effects estimated for the full sample. The policy implications brought by these effects highlight the fragility of the CEE economies, in case of a slowdown in industrial activity in the Western EU economies. Therefore, we employ a rollingwindow estimation of the VAR model and compute an overall Industrial Production Spillover Index (the results of the index are presented in Figure 1). We aim to include the index in a VAR forecasting framework in order to test its value in signaling risks related to synchronized increases in production activity which can lead to negative effects on small open economies (such as the CEE region) in case of an economic downturn. 
Global Value Chains and Industrial Production Spillovers: Where Does the CEE Region Stand?

Figure 1. The estimated Spillover Index using the Diebold-Yilmaz methodology

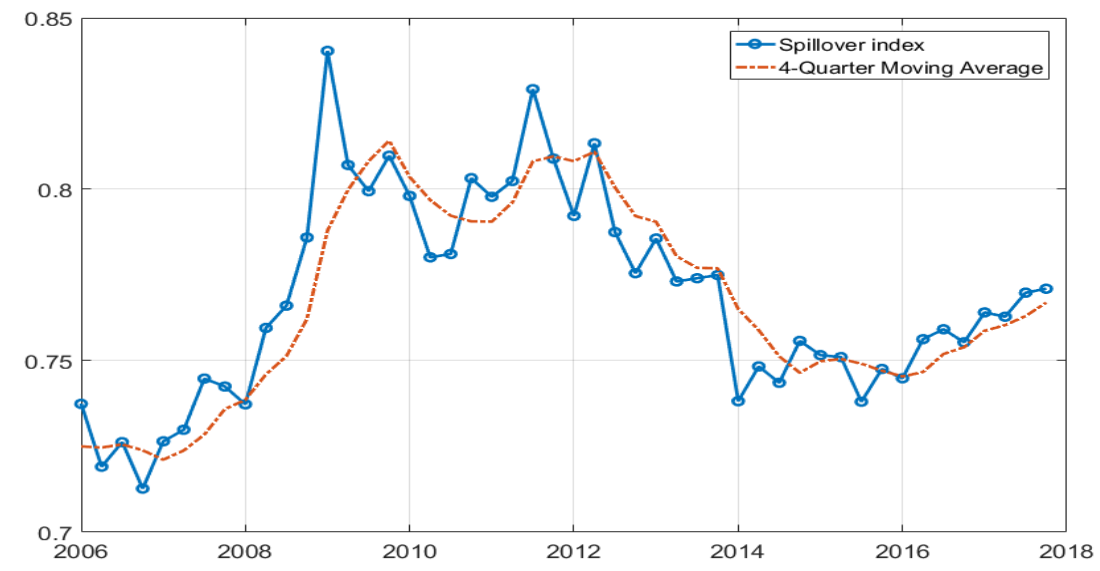

Source: authors' estimation

\section{Can information related to industrial production spillovers improve forecast accuracy? Evidence from a large BVAR model for the CEE economies}

\subsection{The BVAR model}

In the second section of the study, the goal is to argue that the industrial production spillover index can provide insightful information for policymakers and should be taken into account when conducting macroeconomic policy analysis. In order to achieve this goal, we resort to a straightforward medium-sized forecasting model using the well-known VAR framework, estimated using Bayesian techniques. The choice regarding the size of the model is argued by the fact that including the index in a small model could potentially lead to an omitted variable bias, as described in Christiano, Eichenbaum and Evans (1999) or Giannone and Reichlin (2006). Moreover, the risk of overfitting the model using an excessively large dataset is overcome by using Bayesian shrinkage (Bańbura, Giannone and Reichlin, 2008). The authors show that adjusting the tightness of the priors when adding more variables is sufficient to deal with the aforementioned problems.

Formally, for a VAR (p) model:

$$
Y_{t}=c+\sum_{i=1}^{p} A_{i} Y_{t-i}+u_{t}, \text { with } E u_{t} u_{t}^{\prime}=\Psi
$$

Following mainstream literature on this topic, we set the prior beliefs using the methodology proposed by Litterman (1986), which ensures the assumptions that the more recent lags provide more reliable information: 


$$
E\left[\left(A_{k}\right)_{i j}\right]=\left\{\begin{array}{c}
\delta, j=i, k=1 \\
0, \text { otherwise }
\end{array}, \quad \operatorname{VAR}\left[\left(A_{k}\right)_{i j}\right]=\left\{\begin{array}{c}
\frac{\lambda^{2}}{k^{2}}, j=i \\
v \frac{\lambda^{2}}{k^{2}} \frac{\sigma_{i}^{2}}{\sigma_{j}^{2}}, \text { otherwise }
\end{array}\right.\right.
$$

The key hyperparameter used to deal with the issue of overfitting is $\lambda$, which controls the overall tightness of the $a$ priori distribution. If $\lambda$ is close to zero then the posterior distributions are equal to the priors i.e. the data has no effect on the results, while for a very large value of $\lambda$ the posterior results are close to the OLS estimates. One additional solution for choosing the optimal hyperparameter values is given by Giannone et al. (2012), by estimating the hyperparamenters within the Bayesian framework. The authors propose setting the values that maximize the marginal likelihood, for all given combination of parameters by running a grid search.

The practical implementation of the prior distribution takes the form proposed by Kadiyala and Karlsson (1997) and Robertson and Tallman (1999), through the Normal Inverted Wishart prior:

$$
\operatorname{vec}(B) \mid \Psi \sim N\left(\operatorname{vec}\left(B_{0}\right), \Psi \otimes \Omega_{0}\right) \text { and } \Psi \sim i W\left(S_{0}, \alpha_{0}\right)
$$

The prior distributions are implemented using dummy observations that match the Minnesota moments (for more details regarding the technical implementations see, for example,Dieppe et al. 2016). It has been shown that the priors can be written in terms of matrices $Y_{d}$ and $X_{d}$ where:

$$
\begin{gathered}
Y_{d}=\left(\begin{array}{c}
\operatorname{diag}\left(\delta_{1} \sigma_{1}, \ldots, \delta_{n} \sigma_{n}\right) / \lambda \\
0_{n(p-1) \times n} \\
\ldots \\
\operatorname{diag}\left(\sigma_{1}, \ldots, \sigma_{n}\right) \\
0_{1 \times n}
\end{array}\right), \\
X_{d}=\left(\begin{array}{cc}
J_{p} \otimes \operatorname{diag}\left(\sigma_{1}, \ldots, \sigma_{n}\right) / \lambda & 0_{n p \times 1} \\
\ldots & \ldots \\
0_{n \times n p} & 0_{n \times 1} \\
\ldots & \ldots \\
0_{1 \times n p} & \epsilon
\end{array}\right)
\end{gathered}
$$

With $B_{0}=\left(X_{d}^{\prime} X_{d}\right)^{-1} X_{d}^{\prime} Y_{d}, \Omega_{0}=\left(X_{d}^{\prime} X_{d}\right)^{-1}$ and $S_{0}=\left(Y_{d}-X_{d} B_{0}\right)^{\prime}\left(Y_{d}-\right.$ $\left.X_{d} B_{0}\right)$, on which we impose the Normal Inverse distribution described above. Bańbura, Giannone and Reichlin(2008) intuitively explain that the first block of dummies is related to the autoregressive coefficients, the second reflects the covariance matrix and the last block corresponds to the intercept.

The BVAR model is estimated using MCMC techniques, namely Gibbs sampling, using 10,000 simulations and a burn-in of 8,000 iterations. For a detailed description of the MCMC algorithm, please see Blake and Mumtaz (2002) for an excellent in-depth review of Bayesian econometrics. In the context, of Bayesian estimation, density forecasting is a straightforward technique implemented within 
Global Value Chains and Industrial Production Spillovers: Where Does the CEE Region Stand?

the Gibbs sampling algorithm. It makes use of the simulations drawn for the parameters and covariance matrix, as described above, to generate recursively the simulated values of the endogenous variables. Through this iterative algorithm, we obtain a sample of independent draws from the joint predictive distribution.

The final part of the analysis implies comparing forecasting accuracy between a baseline model which does not incorporate any information related to industrial production spillovers and the augmented model which includes the spillover index. For this part, we have chosen to compute two alternative density forecast evaluation measures: the Log Predictive Score (LPS) and the Cumulative Ranked Predictive Score (CRPS). To provide further evidence, we use the Log Predictive Score as an error measure function to compute the forecasting accuracy test formulated by Giacomini and White (2006).

The empirical literature contains several formulations for the log predictive score (for example, see Mao, 2010 or Warne et al., 2013), with slight adjustments that do not change the overall interpretation of the indicator. Formally, the log predictive score for a given horizon $(h)$ is determined by:

$$
\operatorname{LPS}\left(Y_{t}\right)=\log f\left(Y_{t+1: t+h} \mid Y_{t}\right)
$$

Intuitively, the score denotes the forecast density, at horizon $h$, evaluated at the realized data point. A larger log score indicates the model produces a more accurate forecast density. An alternative indicator to the LPS is the Continuous Ranked Probability Score, introduced by Matheson and Winkler (1976) and popularized more recently by Gneiting and Raftery (2007). The idea behind the indicator is similar to the LPS, but extended to incorporate information related to the sharpness of the forecast density around the realized value. If $F$ is the cumulative distribution function corresponding to the marginal forecast density, then the CRPS is defined as:

$$
\operatorname{CRPS}\left(F, Y_{t+h}\right)=\int_{-\infty}^{\infty}\left(F(x)-1_{\left(x>Y_{T+h}\right)}\right)^{2} d x
$$

Where $1_{\left(x>Y_{T+h}\right)}$ is an indicator function which takes the value 1 when the condition is fulfilled. Taking the form of a penalty function, it takes the ideal value 0 in the case of a perfect density distribution, respectively a positive and larger value, the poorer the model forecast accuracy is.

The Giacomini-White conditional predictive ability test relies on a loss function, defined for a forecasting model (in our case, we rely on the Log Density Score), to ascertain whether two forecasts are equally accurate on average. Formally, it defines the following null hypothesis:

$$
H_{0}: E\left[L_{t+\tau}\left(Y_{t+\tau}, f_{m, t}\right)-L_{t+\tau}\left(Y_{t+\tau}, g_{m, t}\right) \mid \mathcal{F}_{t}\right]=E\left[\Delta L_{m, t+\tau} \mid \mathcal{F}_{t}\right]=0 \text { a.s } .
$$




\section{Matei Kubinschi, Dinu Barnea}

Some of the benefits of the conditional forecast accuracy tests are their unified treatment of nested and non-nested tests and the fact that they do not impose restrictions on the forecast estimation procedures (Giacomini and White, 2006).

\subsection{Dataset}

For this empirical exercise we focus our attention on 4 countries from the CEE region, namely Romania, Hungary, Poland and the Czech Republic, for which we assess whether adding information related to industrial production spillovers can improve forecasting performance for GDP growth. We build a quarterly dataset starting from 2005 which includes macroeconomic and financial variables such as: real GDP growth, Gross Fixed Capital Formation (GFCF), the Harmonized Index of Consumer Prices (HICP), number of occupied persons, Real Effective Exchange Rate (REER) and a measure for short-term interest rates (3M interbank rates), but also adds some variables related to economic sentiment (the Economic Sentiment Index or ESI) and sectoral indices related to construction, manufacturing and industry orders.

Finally, we augment the model with the spillover index computed in the first part of the study. The series were extracted from the Eurostat databaseand transformed to yearly growth rates, as we are not particularly concerned with stationarity in the context of a Bayesian estimation framework.

\subsection{Calibration and Results}

Mainstream literature on Bayesian VAR estimation using Litterman or "dummy-initial-observation" priors recommend calibrating the shrinkage hyperparameter $\lambda$, which controls the overall tightness, to decreasingly smaller values in direct relation to the increase in the size of the model in order to avoid overfitting (see, for example, Giannone, Lenza and Primiceri, 2012). Although we argue that the model is a medium size one, with 11 variables, we ensure the robustness of the results by estimating the model using a range of strategies and comparing the results based on the forecasting accuracy indicators.

We estimate the model iteratively on 16 quarters, and compute density forecasts for 1 to 8 steps-ahead, corresponding to a 2-year horizon for predicting the real GDP growth rate. In this case, we are limited by the relatively short macroeconomic and financial time series available for the selected CEE economies. Figure 2 illustrates an example of the results for the density forecasting using the augmented BVAR model, calibrated using the value for the shrinkage parameter which corresponds to the lowest forecast error. Due to the fact that we compute the average value for the forecast error, in this case the Continuous Ranked Probability Score (CRPS), the forecasts will provide the best fit along the 8 -quarter horizon, not focusing on either the short or long-term out-of-sample forecast accuracy. 
Global Value Chains and Industrial Production Spillovers: Where Does the CEE Region Stand?

\section{Figure 2. Density forecast for real GDP growth on $8 Q$ horizon (2014-2016)}
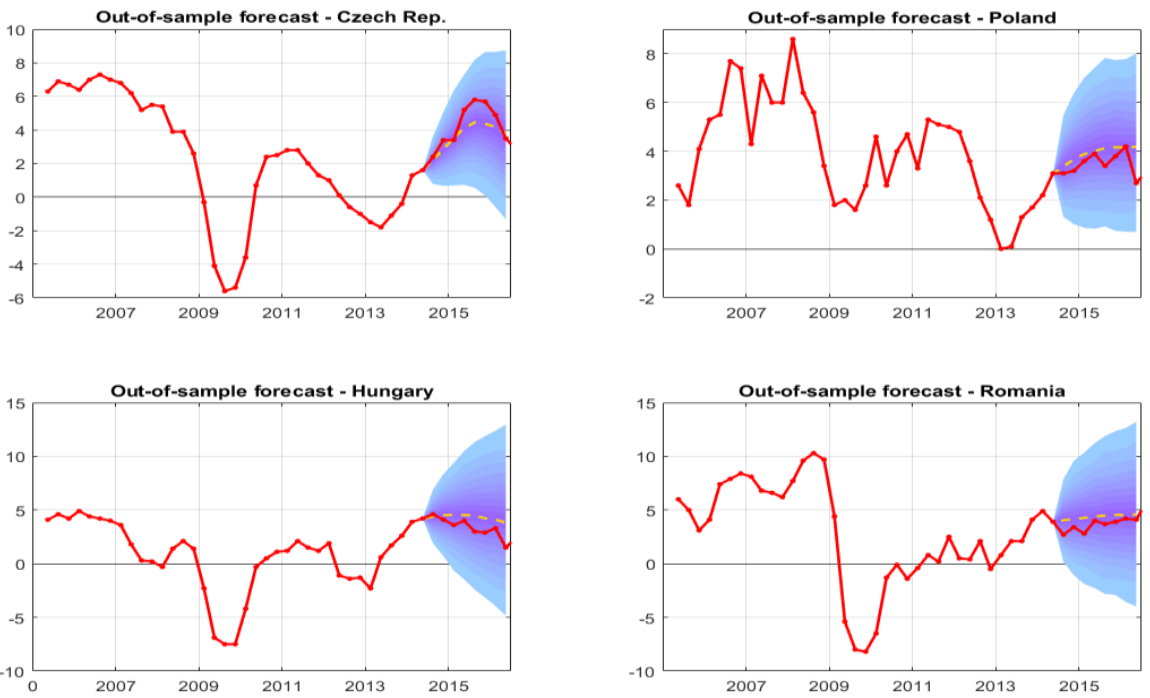

Note: the shrinkage parameter was chosen by the optimisation method described at the beginning of the section, by running a grid search and setting the value which minimises the CRPS indicator; the dotted line represents the median of the forecast density, while the shaded blue areas illustrate the 5 95 quantiles of the forecast density.

Source: authors' estimation

In the first and second approaches, we set the hyperparameter to a fixed value across the entire sample using $\lambda=0.1$ and $\lambda=0.4$ while in the third approach we use a grid search for $\lambda \in[0.1 ; 1]$ and choose the optimal value for the parameter which maximizes the log-likelihood function (LLF). In the final setup, we adopt a similar grid search strategy and estimate the model recursively, taking the optimal value for lambda according to the smallest value for the forecasting accuracy indicators, in this case the minimum average value for the CRPS across the forecast horizon

Table 3 presents the results of the calibration strategies for the 2 competing models, excluding and including the Industrial Production Spillover Index. The values computed for each horizon are simple averages for the 16-quarter estimation timeframe. For the first two calibration methods, the hyperparameter value is fixed while for the third and fourth it is estimated within the model and optimally chosen at each iteration. It is therefore straightforward to find comparable results for cases in which the values of the calibrated shrinkage parameter are similar for different methods. 
Table 3. CRPS indicator results for the 4 CEE countries, using multiple prior calibration strategies

\begin{tabular}{|c|c|c|c|c|c|c|c|c|}
\hline \multirow{2}{*}{$\frac{\text { CZE }}{\text { Spillover }}$} & \multicolumn{2}{|c|}{$\lambda=0.1$} & \multicolumn{2}{|c|}{$\lambda=0.4$} & \multicolumn{2}{|c|}{$\lambda=\min (\mathrm{CRPS})$} & \multicolumn{2}{|c|}{$\lambda=\max ($ LLF $)$} \\
\hline & No & Yes & No & Yes & No & Yes & No & Yes \\
\hline 1 & 0.445 & 0.450 & 0.461 & 0.459 & 0.427 & 0.410 & 0.441 & 0.425 \\
\hline 2 & 0.785 & 0.793 & 0.838 & 0.806 & 0.766 & 0.720 & 0.800 & 0.779 \\
\hline 3 & 1.088 & 1.098 & 1.152 & 1.074 & 1.018 & 0.941 & 1.086 & 1.074 \\
\hline 4 & 1.423 & 1.431 & 1.476 & 1.375 & 1.224 & 1.130 & 1.403 & 1.389 \\
\hline 5 & 1.649 & 1.649 & 1.715 & 1.580 & 1.391 & 1.266 & 1.664 & 1.619 \\
\hline 6 & 1.724 & 1.714 & 1.728 & 1.609 & 1.433 & 1.327 & 1.722 & 1.666 \\
\hline 7 & 1.686 & 1.688 & 1.579 & 1.483 & 1.365 & 1.305 & 1.600 & 1.556 \\
\hline 8 & 1.604 & 1.590 & 1.345 & 1.261 & 1.228 & 1.180 & 1.382 & 1.307 \\
\hline avg. & 1.301 & 1.302 & 1.287 & 1.206 & 1.107 & 1.035 & 1.262 & 1.227 \\
\hline
\end{tabular}

\begin{tabular}{|c|c|c|c|c|c|c|c|c|}
\hline POL & \multicolumn{2}{|c|}{$\lambda=0.1$} & \multicolumn{2}{|c|}{$\lambda=0.4$} & \multicolumn{2}{|c|}{$\lambda=\min ($ CRPS) } & \multicolumn{2}{|c|}{$\lambda=\max ($ LLF) } \\
\hline Spillover & No & Yes & No & Yes & No & Yes & No & Yes \\
\hline 1 & 0.576 & 0.573 & 0.754 & 0.745 & 0.562 & 0.577 & 0.589 & 0.869 \\
\hline 2 & 1.029 & 1.024 & 1.383 & 1.385 & 0.962 & 0.992 & 1.038 & 1.543 \\
\hline 3 & 1.307 & 1.294 & 1.823 & 1.848 & 1.195 & 1.223 & 1.304 & 1.964 \\
\hline 4 & 1.515 & 1.511 & 2.152 & 2.173 & 1.378 & 1.405 & 1.513 & 2.291 \\
\hline 5 & 1.516 & 1.507 & 2.071 & 2.092 & 1.334 & 1.357 & 1.492 & 2.084 \\
\hline 6 & 1.406 & 1.414 & 1.798 & 1.820 & 1.234 & 1.228 & 1.378 & 1.713 \\
\hline 7 & 1.294 & 1.315 & 1.458 & 1.480 & 1.114 & 1.104 & 1.248 & 1.335 \\
\hline 8 & 1.139 & 1.162 & 1.119 & 1.140 & 0.986 & 0.972 & 1.099 & 1.036 \\
\hline avg. & 1.223 & 1.225 & 1.570 & 1.585 & 1.096 & 1.107 & 1.208 & 1.604 \\
\hline
\end{tabular}

\begin{tabular}{|c|c|c|c|c|c|c|c|c|}
\hline HUN & \multicolumn{2}{|c|}{$\lambda=0.1$} & \multicolumn{2}{|c|}{$\lambda=0.4$} & \multicolumn{2}{|c|}{$\lambda=\min ($ CRPS) } & \multicolumn{2}{|c|}{$\lambda=\max ($ LLF) } \\
\hline Spillover & No & Yes & No & Yes & No & Yes & No & Yes \\
\hline 1 & 0.655 & 0.642 & 0.773 & 0.776 & 0.617 & 0.618 & 0.788 & 0.730 \\
\hline 2 & 0.968 & 0.946 & 1.164 & 1.152 & 0.774 & 0.774 & 1.241 & 1.126 \\
\hline 3 & 1.259 & 1.235 & 1.456 & 1.406 & 0.845 & 0.827 & 1.683 & 1.405 \\
\hline 4 & 1.643 & 1.611 & 1.802 & 1.718 & 1.084 & 1.032 & 2.112 & 1.747 \\
\hline 5 & 1.764 & 1.735 & 2.044 & 1.924 & 1.230 & 1.171 & 2.383 & 2.101 \\
\hline 6 & 1.914 & 1.895 & 2.315 & 2.215 & 1.502 & 1.431 & 2.556 & 2.509 \\
\hline 7 & 1.995 & 1.979 & 2.451 & 2.387 & 1.779 & 1.714 & 2.613 & 2.755 \\
\hline 8 & 1.989 & 1.969 & 2.511 & 2.468 & 1.967 & 1.926 & 2.562 & 2.785 \\
\hline & 1.523 & .502 & 814 & 1.756 & 1.225 & 1.187 & 1.992 & 1.895 \\
\hline
\end{tabular}

\begin{tabular}{ccccccccc} 
ROM & \multicolumn{2}{c}{$\lambda=0.1$} & \multicolumn{4}{c}{$\lambda=0.4$} & \multicolumn{4}{c}{$\lambda=\min (\mathrm{CRPS})$} & $\lambda=\max ($ LLF) \\
\hline Spillover & No & Yes & No & Yes & No & Yes & No & Yes \\
\hline 1 & 0.758 & 0.755 & 0.756 & 0.805 & 0.735 & 0.751 & 0.825 & 0.929 \\
2 & 0.965 & 0.954 & 1.097 & 1.121 & 0.929 & 0.940 & 1.287 & 1.324 \\
3 & 1.100 & 1.096 & 1.477 & 1.491 & 1.099 & 1.103 & 1.775 & 1.826 \\
4 & 1.219 & 1.201 & 1.690 & 1.716 & 1.177 & 1.182 & 2.115 & 2.131 \\
5 & 1.231 & 1.218 & 1.666 & 1.663 & 1.208 & 1.200 & 2.252 & 2.185 \\
6 & 1.275 & 1.277 & 1.610 & 1.602 & 1.257 & 1.253 & 2.265 & 2.202 \\
7 & 1.314 & 1.317 & 1.540 & 1.552 & 1.320 & 1.316 & 2.338 & 2.264 \\
8 & 1.412 & 1.400 & 1.594 & 1.609 & 1.402 & 1.399 & 2.380 & 2.361 \\
\hline avg. & 1.159 & 1.152 & 1.429 & 1.445 & 1.141 & 1.143 & 1.905 & 1.903 \\
\hline
\end{tabular}

Source: authors' estimation

First of all, as expected, the smallest error is found for the calibration method which implies choosing the shrinkage parameter which minimizes the CRPS indicator, while the largest error is found for the maximum likelihood method, with the exception of the Czech Republic, where the values for $\lambda$ are similar for the 2 methods.

Secondly, when comparing the forecast accuracy of the two specifications we find that including the Industrial Production Spillover Index provides better forecasting accuracy, on average, for Hungary, confirmed by all the calibration strategies, followed by the Czech Republic and Romania, where the results are mostly positive in favor of including the index but also depend on the choice of the hyperparameter. Conversely, the results for Poland show that the augmented model offers lower forecast accuracy, in all the considered cases. Moreover, the differences in forecast errors, measured by the CRPS, are the largest for Hungary, followed by the Czech Republic and Romania.

In Romania's case, when simulating the optimal value of the shrinkage parameter we find a relatively flat behavior, with the lowest values registered for small values of $\lambda$ (around 0.1 ) or high values (around or even above 1). Therefore, the results are close in all the considered cases, with positive results for the calibration of $\lambda=0.1$ and $\lambda=\max (L L F)$, which in this case is equal to 1 . The situation is somewhat similar in the case of the Czech Republic where we find positive results for the augmented model when the shrinkage parameter comes close to the optimum value computed using the CRPS criterion. In turn, setting lower values leads to a lower forecast accuracy of the augmented model. 
Global Value Chains and Industrial Production Spillovers: Where Does the CEE Region Stand?

In Poland's case, we find significant differences between the results of the grid search for the third and fourth calibration methods. Nevertheless, in any of the considered calibration strategies, the results demonstrate that the augmented model has a lower forecasting performance.

\section{Figure 3. Median CRPS values for the model specifications using the 4 calibration methods for the shrinkage parameter}
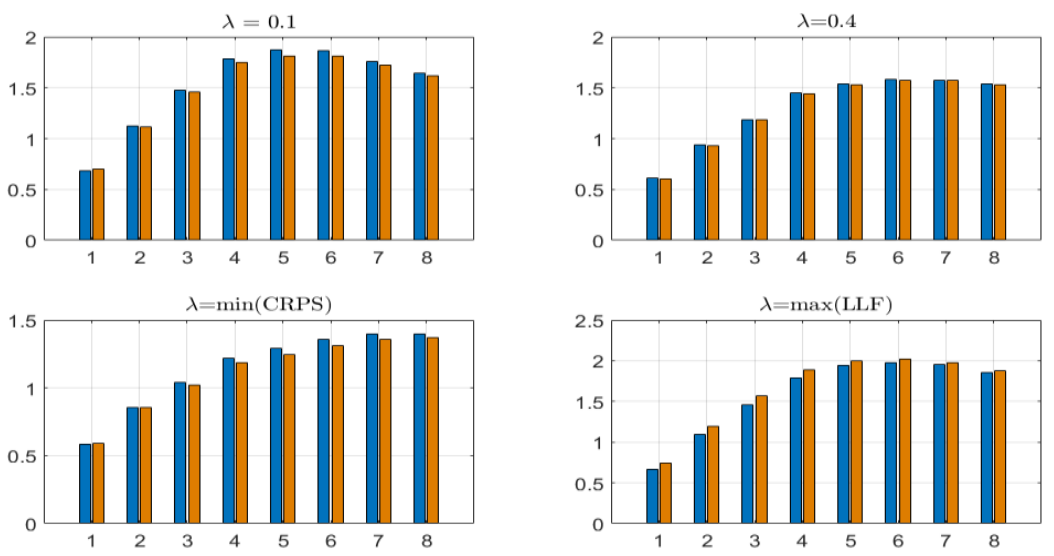

Note: the blue bars represent the CRPS average value across all countries for the model excluding the Industrial Production Spillover Index while the orange bars represent the results of the augmented model, for a given time horizon

Source: authors' estimation

Aggregating the results by calibration method highlights once again the fact that, in the case of a grid search for the optimum value of the shrinkage parameter, we find that the augmented model produces more accurate forecast results (Figure 3). The argument is true for the fixed calibration as well, while setting the hyperparameter to the value which maximizes the LLF, leads to lower average forecast accuracy for the augmented model which includes the spillover index. Analogous conclusions can be drawn from analyzing the results for the Log Density Score averages. Finally, when taking into account the time horizon, we find that in general the gains in forecast accuracy are medium or long-term, highlighting the importance of a timely identification of a build-up of excessive spillovers, which can lead to a downturn, and consequently to a proper downward adjustment of growth perspectives.

\subsection{Robustness check}

In the previous section, we have provided strong evidence to support the fact that including the Industrial Production Spillover Index in a medium-sized VAR model improves forecasting accuracy for Hungary, followed by the Czech 
Republic and Romania. To achieve this goal, we proposed a set of four calibration techniques and provided forecast evaluation indices for all of these methods.

To further support our conclusions, we present the results obtained for the forecast evaluation indices described in the methodology section. In Figure 4 we present the Log Density Score (LDS) obtained for each country in the 16-quarter timeframe and on an 8-quarter horizon.

Figure 4. Log Density Score (LDS) results for the Density forecasts, on an $8 Q$ horizon (2012-2015)
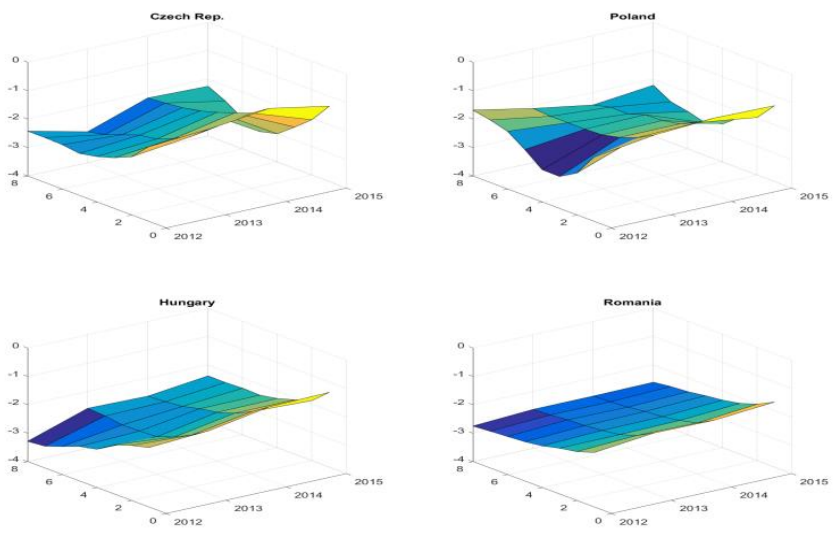

To get a better grasp of the augmented model forecasting performance, we plot the 1-step-ahead median forecast versus the realized rate of real GDP growth for all the countries analyzed in the second part of the study (Figure 5). A visual analysis confirms the performance of the results for the 3 countries identified using the results provided in Table 3, while in the case of Poland, a relatively wide deviation from the realized values can be easily observed.

Figure 5. 1-step-ahead median forecast versus realized real GDP growth values
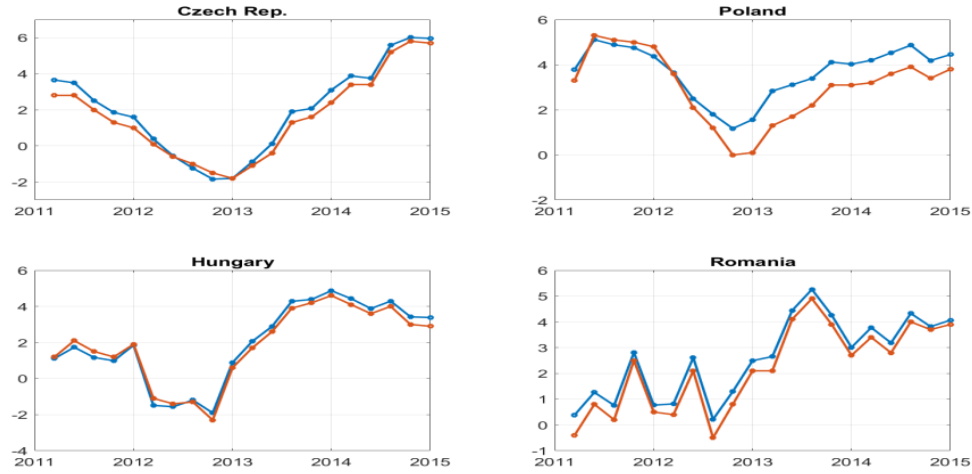
Global Value Chains and Industrial Production Spillovers: Where Does the CEE Region Stand?

Finally, we provide further evidence via statistical testing, using the Giacomini-White Test of Conditional Predictive Ability (Table 4). The main drawback in this case is the limited time frame available for inference which can be problematic for the power of the test. Therefore, we choose to report the results of the test as further additional evidence to support our findings, rather than base the conclusions solely on the results of the testing procedure.

We compute the test statistic using the Log Density Score (LDS) results presented above, as the loss function required by the testing framework, in order to account for the features of the density forecasting methodology. The test is built by extracting samples on each time horizon (from 1Q to 8Q) and for each country and testing the null hypothesis defined in section 4.1. In order to illustrate the differences highlighted in the previous section related to calibration strategies, we report the results for the test under two of the four calibration approaches: the fixed value calibration $(\lambda=1)$ and the optimization using the CRPS criterion.

Table 4. Results for the Giacomini-White Test, for different calibration of shrinkage parameter

\begin{tabular}{|c|c|c|c|c|c|c|c|c|c|c|c|c|c|c|c|c|c|}
\hline & \multicolumn{8}{|c|}{$\lambda=\min (C R P S)$} & \multicolumn{9}{|c|}{$\lambda=0.1$} \\
\hline & \multicolumn{2}{|c|}{ Czech Rep. } & \multicolumn{2}{|c|}{ Poland } & \multicolumn{2}{|c|}{ Hungary } & \multicolumn{2}{|c|}{ Romania } & \multicolumn{3}{|c|}{ Czech Rep. } & \multicolumn{2}{|c|}{ Poland } & \multicolumn{2}{|c|}{ Hungary } & \multicolumn{2}{|c|}{ Romania } \\
\hline Horz. & Sign & T-stat. & Sign & T-stat. & Sign & T-stat. & Sign & T-stat. & Horz & z. Sign & T-stat. & Sign & T-stat. & Sign & T-stat. & Sign & T-stat. \\
\hline 1 & $(+)$ & 2.36 & $(-)$ & 0.89 & $(-)$ & 0.32 & $(+)$ & 0.04 & 1 & $(-)$ & 0.01 & $(+)$ & 1.63 & $(+)$ & $4.89 * *$ & $(+)$ & 2.54 \\
\hline 2 & $(+)$ & $2.77^{*}$ & $(-)$ & 0.65 & $(+)$ & $3.78^{*}$ & $(+)$ & 1.62 & 2 & $(+)$ & 0.11 & $(+)$ & 0.59 & $(+)$ & $3.80^{*}$ & $(+)$ & 1.09 \\
\hline 3 & $(+)$ & $3.00 *$ & $(-)$ & 0.59 & $(+)$ & $6.52^{* *}$ & $(+)$ & $5.65^{* *}$ & 3 & $(+)$ & 0.49 & $(+)$ & 0.63 & $(+)$ & $3.63 *$ & $(+)$ & $3.61^{*}$ \\
\hline 4 & $(+)$ & $4.55^{* *}$ & $(-)$ & 0.62 & $(+)$ & $5.39 * *$ & $(+)$ & 0.01 & 4 & $(+)$ & 0.92 & $(+)$ & 0.19 & $(+)$ & $3.38^{*}$ & $(+)$ & $2.94^{*}$ \\
\hline 5 & $(+)$ & $10.25^{* * *}$ & $(-)$ & 0.01 & $(+)$ & $3.98 * *$ & $(+)$ & 0.13 & 5 & $(+)$ & 1.73 & $(+)$ & $9.70 * * *$ & $(+)$ & $3.28 *$ & $(+)$ & 1.59 \\
\hline 6 & $(+)$ & $15.48 * * *$ & $(+)$ & 1.13 & $(+)$ & $4.16^{* *}$ & $(-)$ & 0.02 & 6 & $(+)$ & 1.44 & $(+)$ & $4.33^{* *}$ & $(+)$ & $5.00 * *$ & $(+)$ & $3.54^{*}$ \\
\hline 7 & $(+)$ & $4.485^{* *}$ & $(+)$ & 1.11 & $(+)$ & $7.08^{* * *}$ & $(+)$ & 1.19 & 7 & $(+)$ & 0.50 & $(+)$ & 0.91 & $(+)$ & $4.22^{* *}$ & $(+)$ & $3.63^{*}$ \\
\hline 8 & $(+)$ & 2.55 & $(+)$ & 1.21 & $(+)$ & $8.39 * * *$ & $(+)$ & 1.34 & 8 & $(+)$ & 0.16 & $(-)$ & 1.71 & $(+)$ & $5.03 * *$ & $(+)$ & $20.00^{* * *}$ \\
\hline
\end{tabular}

Note: $(+)$ indicates the fact that the second model (augmented with the industrial production spillover index provides better forecasting accuracy while (-) indicates that the baseline model is more accurate

\section{Source: authors' estimation}

Turning to the results, we find evidence that strongly support our initial findings. First of all, the highest test-statistics, corresponding to a better performance of the augmented model, were found for Hungary. Moreover, in the case of Hungary, we find the most cases with statistically significant forecast horizons. Additionally, we find stronger statistical significance for the longer forecast horizons, once again supporting our claim that including the Industrial Production Spillover Index improves forecast accuracy especially in the medium term.

Secondly, the results for the Czech Republic and Romania depend on the calibration approach: for the Czech Republic we find positive results regarding the augmented model in case of the optimization via CRPS indicator while for 


\section{Matei Kubinschi, Dinu Barnea}

Romania, the positive results are obtained when choosing the fixed, small value for the hyperparameter. Again, this is in accordance to the aforementioned results from Table 3. Finally, we find evidence of negative performance in case of Poland, for the optimization calibration strategy, but the test statistics are not large enough to formulate a robust conclusion regarding the superior model, in this case.

\section{Conclusions}

The main objective of the paper was to develop a better understanding of how the industrial sectors in different countries influence each other and how developments on western markets impact the growth rates of emerging economies.

By applying the Diebold-Yilmaz spillover index methodology, we find that after the 2009-2011 downturn, individual contributions of each country's industrial production index now play a much smaller part in explaining overall dynamics, partially confirming the fact that spillovers from other countries have become increasingly important in recent years. This effect is especially strong in the CEE region, where we observe a significant increase in spillovers from Germany, France, Portugal and the Netherlands which now account, in some cases, for almost double the effects estimated for the entire sample. As a result, we employ a rolling-window estimation of the VAR model and compute an overall Industrial Production Spillover Index and assess whether augmenting a medium-scale VAR model with industrial spillover information can lead to an increase in forecasting accuracy.

In the second part of the analysis, we build a rigorous BVAR estimation framework, with multiple calibration strategies, for the Czech Republic, Poland Hungary and Romania and evaluate the gains or losses from including the Industrial Production Spillover Index in the forecasting framework. We find that adding information related to industrial production spillovers provides better forecasting accuracy, on average, for Hungary, confirmed by all the calibration strategies, followed by the Czech Republic and Romania, where the results are mostly positive in favor of including the index but also depend on the choice of the calibration methodology. Conversely, the results for Poland show that the augmented model offers lower forecast accuracy, in all the considered cases. Moreover, the negative differences in forecast errors are the largest for Hungary, followed by the Czech Republic and Romania. Finally, as a robustness check, we provide further evidence via statistical testing, using the Giacomini-White Test of Conditional Predictive Ability (CPA), and largely confirm our results.

From a policy recommendation perspective, we have shown that tracking the dynamics of the industrial sector without taking into account spillover and contagion effects can lead to significant underestimation of structural vulnerabilities and, consequently, to suboptimal policy decisions. Therefore, policymakers should assess whether information related to industrial sector spillovers are relevant for their economy and potentially include this information as part of the overall framework for monitoring macroeconomic policy. 
Global Value Chains and Industrial Production Spillovers: Where Does the CEE Region Stand?

\section{REFERENCES}

[1]Bańbura, M., Giannone, D. and L. Reichlin (2008), Large Bayesian VARs; Working Papers ECARES 2008_033, ULB ; Universite Libre de Bruxelles;

[2]Blake, S. and H. Mumtaz (2002), Applied Bayesian Econometrics for Central Bankers; Centre for Central Banking Studies, Bank of England;

[3]Christiano, L. J., Eichenbaum, M. and C. L. Evans (1999), Monetary Policy Shocks: What Have We Learned and to What End?, in J. B. Taylor \& M. Woodford (ed.), Handbook of Macroeconomics, edition 1, volume 1, chapter 2, pages 65-148, Elsevier;

[4]Diebold, F. and K. Yilmaz (2009), Measuring Financial Asset Return and Volatility Spillovers, with Application to Global Equity Markets; Economic Journal, Royal Economic Society, vol. 119(534), pp. 158-171;

[5] Diebold, F. and K. Yilmaz (2012), Better to Give than to Receive: Predictive Directional Measurement of Volatility Spillovers; International Journal of Forecasting, Elsevier, vol. 28(1), pages 57-66;

[6]Dieppe, A., van Roye, B. and R. Legrand (2016), The BEAR Toolbox; Working Paper Series 1934, European Central Bank;

[7]Edwards, S. and R. Susmel (2001), Volatility Dependence and Contagion in Emerging Equity Markets; NBER Working Paper No. 8506;

[8]Engle, R.F., Ito, T. and W. Lin (1990), Meteor Showers or Heat Waves Heteroskedastic Intra-Daily Volatility in the Foreign Exchange Market; Econometrica, 58, 525-542;

[9]Giacomini, R. and H. White (2006), Tests of Conditional Predictive Ability; Econometrica, 74, 1545-1578;

[10]Giannone, D., Lenza, M. and G. Primiceri (2012), Prior Selection for Vector Autoregressions; ECB Working Paper No. 1494;

[11]Giannone, D. and L. Reichlin (2006), Does Information Help Recovering Structural Shocks from Past Observations; Journal of the European Economic Association, MIT Press, vol. 4(2-3), pages 455-465, 04-05;

[12]Gneiting, T. and Raftery, A. (2007), "Strict Proper Scoring Rules, Prediction, and Estimation", Journal of the American Statistical Association, 102(477)

[13] Kadiyala, K. R. and S. Karlsson (1997), Numerical Methods for Estimation and Inference in Bayesian VAR-Models; Journal of Applied Econometrics, 12(2), pp. 99-132;

[14]King, M. A., Sentana, E. and S. Wadhwani(1994), Volatility Links between National Stock Markets; Econometrica, Vol. 62, pp. 901-933;

[15]Koop, G., Pesaran, M. H. and S.M. Potter (1996), Impulse Response Analysis in Non-Linear Multivariate Models; Journal of Econometrics 74: 119-147;

[16]Litterman, R. (1986), A Statistical Approach to Economic Forecasting; Journal of Business and Economic Statistics, 4(1), pp. 1-4. 
Matei Kubinschi, Dinu Barnea

[17]Mao, W. (2010), “Bayesian multivariate predictions”,PhD thesis, University of Iowa.

[18]Pesaran, M.H. and Y. Shin (1998), "Generalized Impulse Response Analysis in Linear Multivariate Models", Economics Letters 58: 17-29

[19]Robertson, J. C. and E. W. Tallman (1999), "Vector autoregressions: forecasting and reality", Economic Review, (Q1), pp. 4-18

[20]Matheson, J. and Winkler, R. (1976), "Scoring Rules for Continuous Probability Distributions", Management Science, 22:1087-1096.

[21]Warne, A., Coenen, G., and K. Christoffel (2013),"Marginalised Predictive Likelihood Comparisonswith Application to DSGE, DSGE-VAR, and VAR Models",Technical report, Center for Financial Studies, 376

[22]Yilmaz, K. (2009), "International Business Cycle Spillovers", Koç UniversityTUSIAD Economic Research Forum Working Papers 0903, Koc UniversityTUSIAD Economic Research Forum, revised Nov 2009. 